\title{
Benefits, advantages, and challenges of dual degree programs and implications for the development of therapy science programs \\ Nutzen, Vorteile und Herausforderungen des dualen Studiums und Implikationen für die Entwicklung therapiewissenschaftlicher Studiengänge
}

\author{
Claudia Winkelmann ${ }^{1 *}$, Andrea Helmer-Denzel ${ }^{2}$, \\ Andrea Bosch ${ }^{3}$, Anke Simon ${ }^{3}$

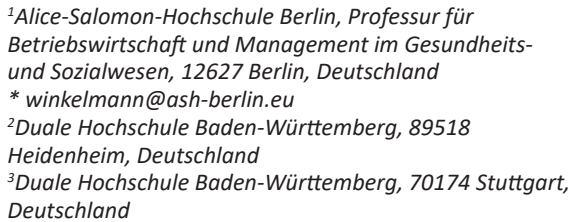

Received 1 May 2021, accepted 1 June 2021

\begin{abstract}
This article introduces the history and philosophy of the Baden-Württemberg Cooperative State University (DHBW). The central aspect is the interlocking of university education with practical companies, which is regulated by clear standards and thus ensures quality and studyability. This interlinking enables a rapid response to changing social requirements, globalization and the digital transformation. The study models, the special roles and challenges for the dual partners, for the study program management and the for students are highlighted. This article is based on the presentation with the same title given at the VFWG symposium, „Are dual courses of study for midwives a model for the therapy professions?" on March 18, 2021.
\end{abstract}

\begin{abstract}
Dieser Beitrag stellt einleitend die Geschichte und die Philosophie der Dualen Hochschule Baden-Württemberg (DHBW) dar. Zentral ist die Verzahnung von hochschulischer Ausbildung mit Praxisbetrieben, die durch klare Standards reglementiert ist und damit Qualität und Studierbarkeit gewährleistet. Diese Verzahnung ermöglicht ein rasches Reagieren auf sich wandelnde gesellschaftliche Anforderungen, die Globalisierung und die digitale Transformation. Für den Studienbereich Gesundheit der DHBW werden die Studienmodelle, die besonderen Rollen und Herausforderungen für die Dualen Partner, für die Studiengangleitung und die Studierenden beleuchtet. Dieser Artikel basiert auf dem Vortrag mit dem gleichen Titel gehalten am VFWG-Symposium «Sind duale Studiengänge der Hebammen ein Modell für die Therapieberufe?» vom 18. März 2021.
\end{abstract}

\section{Keywords}

Baden-Württemberg Cooperative State University - midwives - nursing - therapy professions

\section{Keywords}

Duale Hochschule Baden-Württemberg - Hebammen - Pflege - Therapieberufe

Der Beitrag stützt sich auf die Besonderheiten therapiewissenschaftlicher dualer Studiengänge, die explizit an der Dualen Hochschule Baden-Württemberg (DHBW) zur Bedarfsdeckung im Sozial- und Gesundheitswesen angeboten werden. Dabei werden konkrete Erfahrungen aus verschiedenen Perspektiven präsentiert und in den Kontext des VFWG-Symposiums mit dem Titel „Sind duale Studiengänge der Hebammen ein Modell für die Therapieberufe?“" gestellt.

\section{HISTORIE UND PHILOSOPHIE DER DHBW}

Das duale Studienmodell der DHBW ist auf den Mangel an akademisch ausgebildeten Fachkräften im Zusammenhang mit der prosperierenden Wirtschaft in Baden-Württemberg sowie der Bildungsreform in den 1960er-Jahren zurückzuführen. Einige große Industrieunternehmen wandten sich damals an die Regierung Baden-Württembergs mit Ideen, um den 
Fachkräftemangel in ihren Betrieben durch praxisnahe Studienprogramme zu kompensieren. Tatsächlich hatte ein Betriebsratsvorsitzender den Anstoß gegeben. Eine Grundbedingung war hierbei, dass nicht lediglich Großbetriebe sowie Ballungsgebiete und Universitätsstandorte, sondern alle Regionen und Unternehmen in der Fläche und im ländlichen Raum von den Absolventinnen und Absolventen profitieren sollten. Zudem sollte der Zugang zu Bildung möglichst niedrigschwellig auch für geeignete Interessentinnen und Interessenten aus bildungsfernen Schichten gewährleistet werden. Ein weiteres Erfordernis bestand darin, die Studierenden schnellstmöglich in die betriebliche Praxis zu integrieren. Das war die Geburtsstunde des dualen Konzeptes der Berufsakademien in Baden-Württemberg (vornehmlich bekannt als BA) mit der Verzahnung von Theorie und Praxis auf akademischem Niveau. Als Modellversuch, an dem sich 50 Partnerunternehmen beteiligten, nahmenim Jahr 1974 an zwei Berufsakademien in Stuttgart und Mannheim insgesamt 160 Studierende ihr Studium auf. Im Jahr 2009 wurden die Berufsakademien vom Land Baden-Württemberg hochschulrechtlich anerkannt und in die Duale Hochschule BadenWürttemberg (DHBW) umgewandelt. Die DHBW ist zwischenzeitlich im Landeshochschulgesetz mit ihrem Alleinstellungsmerkmal des dualen Studienmodells mit Verzahnung von Theorie und Praxis explizit aufgenommen. An insgesamt neun Standorten und drei Campus studieren im Jahr 2021 rund 34.000 Studierende aus rund 9000 Partnerunternehmen. Damit ist die staatliche DHBW die größte Hochschule in Baden-Württemberg und der größte Anbieter dualer Studiengänge in Deutschland. Die DHBW bietet ausschließlich duale Studiengänge auf Bachelor- und Master-Niveau und hat ebenfalls mit dem Anspruch der Kooperation mit der Praxis einen spezifischen Forschungsauftrag. Den Rahmen dieses Erfolges bildet das integrierte Qualitätssicherungssystem. Seit dem Jahr 2011 als erste Hochschule in BadenWürttemberg ist die DHBW systemakkreditiert und damit entsprechend berechtigt, ihre Studienangebote selbst zu akkreditieren. Dies ist im Hinblick auf sich ständig wandelnde Anforderungen der Gesellschaft, die digitale Transformation und Globalisierung ein entscheidender Vorteil für die Hochschule und die Ausdifferenzierung der Studienprogramme (DHBW, 2021).

Das duale Modell wurde aufgrund seines Erfolges auch auf die Bundesländer Thüringen und Sachsen ausgeweitet. Hochschulpolitisch unterstützt und mit Programmen gefördert setzte unter anderem in den Ländern Bayern und NRW ein regelrechter Boom der Entwicklung dualer Studienmodelle ein. Der Wissenschaftsrat (WR) nahm dies 2013 zum Anlass, konstituierende Wesensmerkmale des Dualen Studiums in seinem Positionspapier zu definieren und damit einen
Beitrag zur Qualitätssicherung dualer Studienprogramme angesichts zunehmend fehlender Standardisierung und Vergleichbarkeit sowie der heterogenen und sehr einrichtungsspezifischen Interpretation des Terminus „duales Studium“ in Deutschland zu leisten (WR, 2013).

\section{ERWEITERUNG DES STUDIENBEDARFS IM GESUNDHEITSBEREICH}

Der ursprüngliche Bedarf an Fachkräften, die Gewährleistung des breiten Zugangs zu akademischer Bildung und der ausgesprochen große Erfolg des Studienkonzeptes der DHBW waren die Treiber für die Ausweitung der Studienfachbereiche. In BadenWürttemberg hatten die Berufsakademie und die daraus entstandene DHBW einen hervorragenden Ruf. Es lag nahe, dass sich soziale Einrichtungen und Gesundheitseinrichtungen an die Studienakademien und Verantwortlichen in den Ministerien wandten, um über die Studienfachbereiche Wirtschaft, Technik und Sozialwesen hinaus für den Ausbau zu werben. Ab dem Jahr 2012 wurde die Gesundheit gleichberechtigt als vierter Studienfachbereich etabliert. Das duale Studienkonzept bedeutet grundsätzlich, praxisnah und -orientiert sowie primärqualifizierend $\mathrm{zu}$ studieren. Studierende sind nicht lediglich beim Praxisbetrieb angestellt, sondern sie entwickeln Kompetenzen für einen Berufsabschluss und zugleich auch für den ersten akademischen Abschluss.

Insbesondere der Studienfachbereich Gesundheit stellte die DHBW vor große Herausforderungen. Neu waren die Verzahnung des Studiums mit der berufsfachschulischen Ausbildung, die Finanzierung des Studiums durch Praxiseinrichtungen, insbesondere in den Therapieberufen, die akademische Praxis mit entsprechender Anleitung und Betreuung sowie besondere Lehrbedingungen (zum Beispiel Lernkliniken).

Um diese Herausforderungen gemeinsam zu lösen, wurde ein ordentliches Fachgremium eingerichtet, das sich aus Studiendekaninnen und -dekanen, Studiengangleitungen, Studierenden, Vertreterinnen und Vertretern Dualer Partner, der Berufsfachschulen und einem externen Beirat zusammensetzt. Meilensteine in diesem Gremium waren unter anderem die Studienmodell-, die Leitbildund die Prüfungsordnungs-Entwicklung sowie die Weiterentwicklung der Studienprogramme auch im Rahmen der Re-Akkreditierung.

\section{DAS ORIGINAL}

Das Grundmodell der Studienprogramme der DHBW bietet seit dem Jahr 2000 den Bachelor-Grad, der ab dem Jahr 2004 den hochschulrechtlichen Abschlüssen anderer Hochschulen gleichgestellt wurde. Alle Studiengänge 


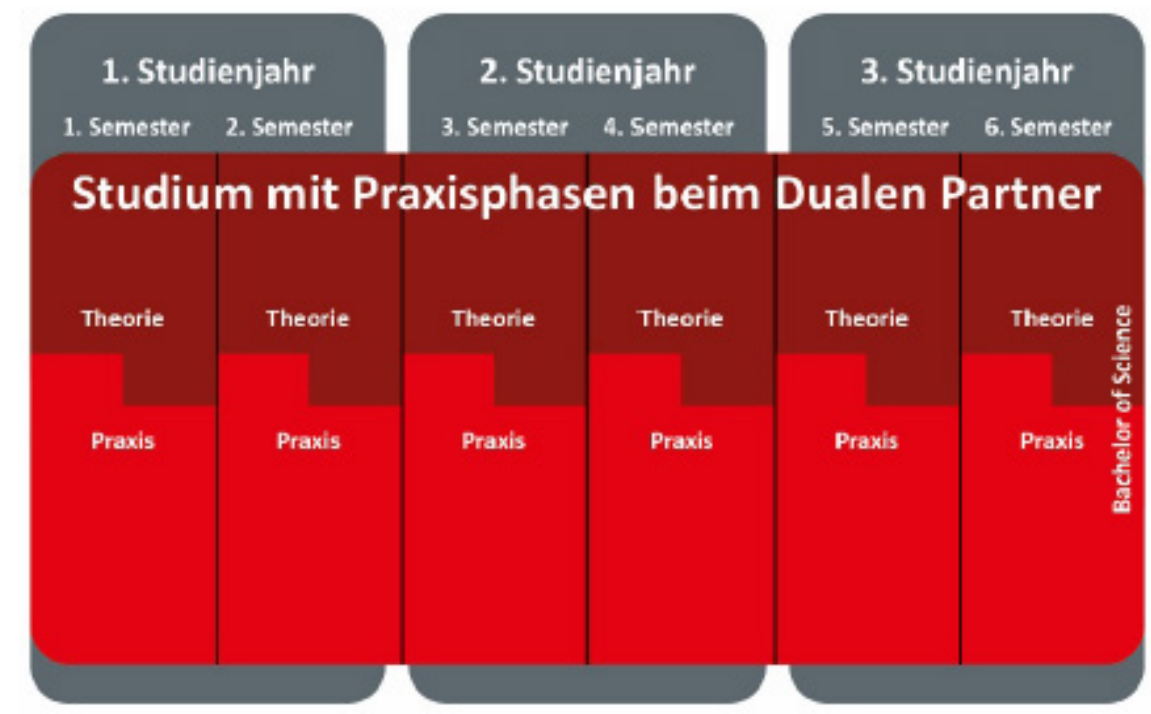

Abbildung 1: Das praxisintegrierende Modell bzw. das Duale Kernmodell im Bereich Gesundheit mit dem akademischen Abschluss Bachelor of Science.

sind als Intensivstudiengänge bewertet. Das bedeutet, der Bachelor-Abschluss umfasst 210 ECTS-Leistungspunkte, die in der Regel innerhalb von 3 Jahren erbracht werden. Unter anderem soll so der Weg der Studierenden zum Master-Abschluss verkürzt werden (DHBW, 2021).

Um die Studierbarkeit im Intensivstudium qualitätsvoll zu ermöglichen, sind an der DHBW folgende Standards etabliert:

- Die Studiengangsleitung betreut die Studierenden in der Theorie- und Praxisphase intensiv. Dies schließt Vor-Ort-Termine bei den Dualen Partnern ein.

- Sie stellt sicher, dass außerdem in der Praxisphase für die Studierenden eine/ ein betriebliche/r Ausbildungsverantwortliche/r im Sinne der Mentorenschaft zur Verfügung steht.

- Die Studiengangsleitung plant die Lehre inkl. der Lernorte anhand eines Modulplans. Ein Lehrbetriebsamt ist nicht vorgesehen. Damit soll eine planbare und verlässliche Studienorganisation sowie Strukturierung des Studiums gewährleistet werden. Die Studiengangsleitung unterstützt die Qualifikation der Lehrenden unter anderem durch Beratung zu den Angeboten des Zentrums für Hochschuldidaktik und lebenslanges Lernen (ZHL) am Center of Advanced Studies (CAS) der DHBW.

- Die Studiengangsleitung ist verantwortlich für die Sicherung der Workload-Verteilung gemäß dem Studienmodell im Studienbereich. Das umfasst auch die im Studienmodell definierte, adäquate Prüfungsdichte, -art und -organisation gemäß Studien- und Prüfungsordnung. Ein zentrales Prüfungsamt ist daher nicht vorgesehen.

- Die Studierenden haben Anwesenheitspflicht.

- Zur Sicherung des Lebensunterhalts der Studierenden und der ausreichenden Erholungszeit gewährt der Duale Partner eine angemessene Vergütung sowie Erholungsurlaub.

Das Grundmodell, das sogenannte Duale Kernmodell, gliedert sich zu 50 Prozent in Praxisphasen beim Dualen Partner und zu 50 Prozent in Theoriephasen an der DHBW, die in der Regel jeweils drei Monate umfassen (s. Abb. 1). Die Gesamtverantwortung obliegt der DHBW. Die „... Duale Hochschule vermittelt durch die Verbindung des Studiums an der Studienakademie mit der praxisorientierten Ausbildung bei den beteiligten Dualen Partnern (duales System) die Fähigkeit zu selbstständiger Anwendung und Weiterentwicklung wissenschaftlicher Erkenntnisse und Methoden in der Berufspraxis. (...) Wer das Studium Sozialpädagogik oder Soziale Arbeit an der DHBW erfolgreich abgeschlossen hat, ist berechtigt, die Berufsbezeichnung ,Staatlich anerkannte Sozialarbeiterin/Sozialpädagogin“ oder ,Staatlich anerkannter Sozialarbeiter/Sozialpädagoge‘ zu führen.“" (LHG, 2020)

\section{ADAPTATION DES DUALEN KERNMODELLS}

\section{AUF DIE GESUNDHEITSSTUDIENGÄNGE}

Die Rahmenstudienmodelle der Studienbereiche der DHBW berücksichtigen die studienbereichsspezifischen Besonderheiten, wie Gesetzgebung, Stakeholder und Infrastruktur, in den jeweiligen Studienangeboten. In einem mehrstufigen Prozess des Studienbereichs wurden und werden hierfür die folgenden Punkte aufeinander abgestimmt: 


\section{CP-Struktur:}

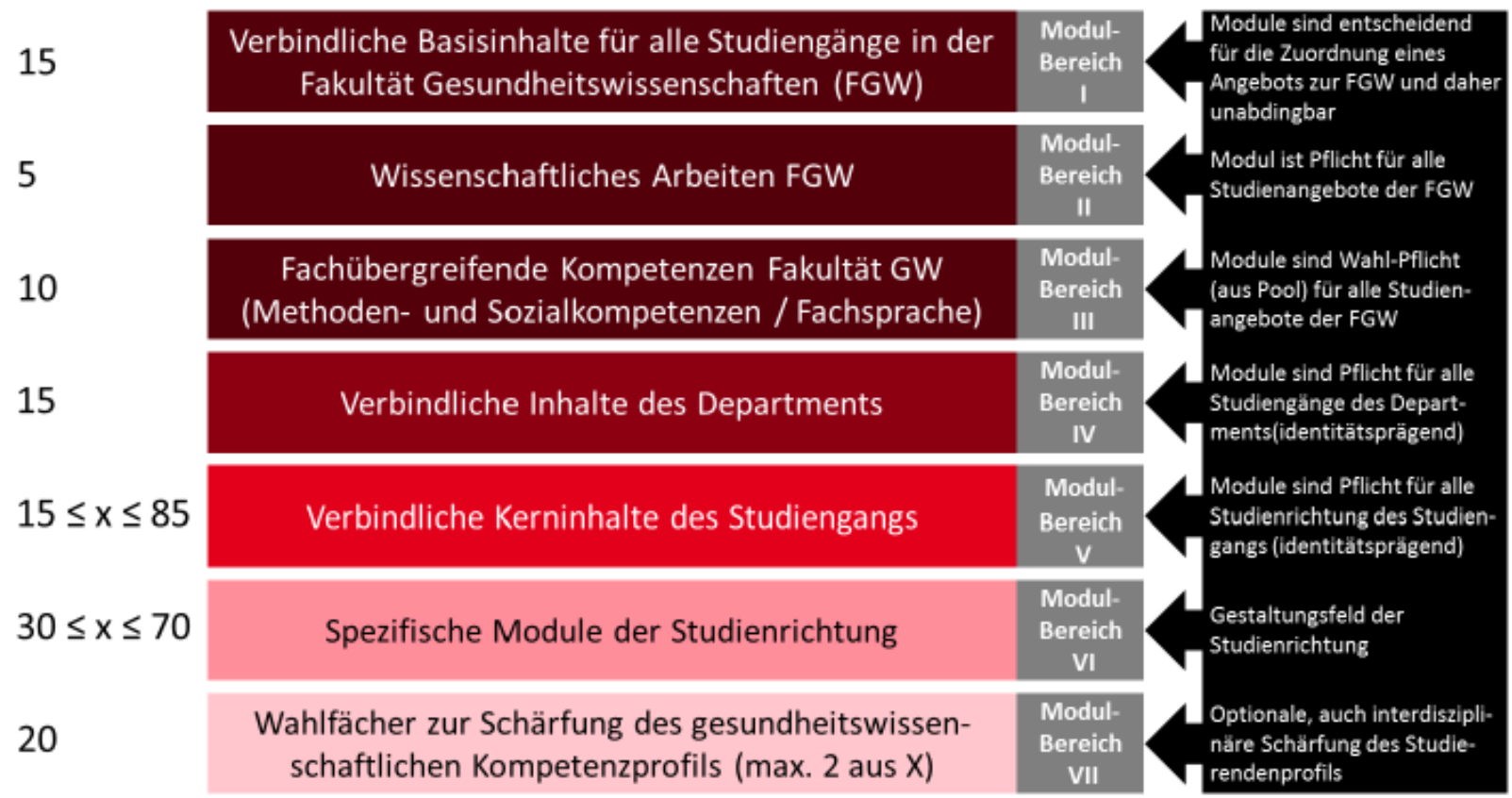

Abbildung 2: Rahmenmodell gesundheitswissenschaftlicher Angebote.

- Struktur der Studiengänge, Studienrichtungen und Schwerpunkte,

- Modularten, Anordnung, Wahlpflicht, studiengangsund standortübergreifende Module,

- Workload-Berechnung und Aufteilung der ECTSLeistungspunkte in den Theorie- und Praxisphasen,

- Prüfungsleistungen und deren Häufigkeit sowie die Bewertungskriterien,

- Relation von Präsenz- und Selbststudienzeit,

- Definition der möglichen Studienformate (Modelle gemäß WR (2013),

- Festlegung der Abschlussbezeichnungen sowie

- Genehmigungsverfahren für die Einrichtung von Vertiefungen etc.

Das Rahmenstudienmodell ist der gemeinsame Strukturierungsrahmen, in dem das Curriculum weiterentwickelt werden kann und ist für alle Studienangebote im Studienbereich Gesundheit verbindlich (s. Abb. 2). Demgemäß wurden alle Gesundheitsstudiengänge entsprechend dieses Modells adaptiert oder bereits dahingehend konzipiert und in einem Re-Akkreditierungsprozess im Jahr 2018 bestätigt. Ziel des inhaltsbezogenen Rahmenmodells ist es, die Gesundheitsstudiengänge von den weiteren Studienangeboten im Portfolio der DHBW abzugrenzen sowie innerhalb des Studienfachbereichs zu vereinheitlichen und beispielsweise auch Synergien oder Kooperations- und Wahlmöglichkeiten zu identifizieren.
Bezogen auf das Studienformat dient das Duale Kernmodell den Gesundheitsstudiengängen als Referenzmodell (s. Abb. 1). Zusätzlich waren die Formate zu definieren, um einerseits dem Fachkräftebedarf der Dualen Partner bestmöglich nachzukommen sowie andererseits die aktuellen in Deutschland geltenden Rahmenbedingungen der Akademisierung in den Gesundheitsfachberufen abbilden zu können. Dafür wurde ein ausbildungsintegrierendes und ein AnrechnungsModell geschaffen (s. Abb. 3 und 4).

\section{DAS PRIMÄRQUALIFIZIERENDE HEBAMMENSTUDIUM IN TRADITION DES DUALEN STUDIUMS AN DER DHBW}

Mit der Reform der Hebammenausbildung zum 1. Januar 2020 wurde erstmals das duale Studium als neue Ausbildungsform und einziger Weg zur Berufszulassung für einen Heilberuf eingeführt. Das duale Hebammenstudium schließt mit der Verleihung des akademischen Grades durch die Hochschule ab und umfasst eine staatliche Prüfung zur Erlangung der Erlaubnis zum Führen der Berufsbezeichnung „Hebamme“ gemäß HebG (2021).

Eine akademische Ausbildung von Hebammen in Deutschland wurde aufgrund einer EU-Richtlinie von 2005, geändert 2013, erforderlich. Der tieferliegende Grund hierfür ist jedoch die zunehmende Komplexität der Anforderungen an die Hebammenarbeit in der 


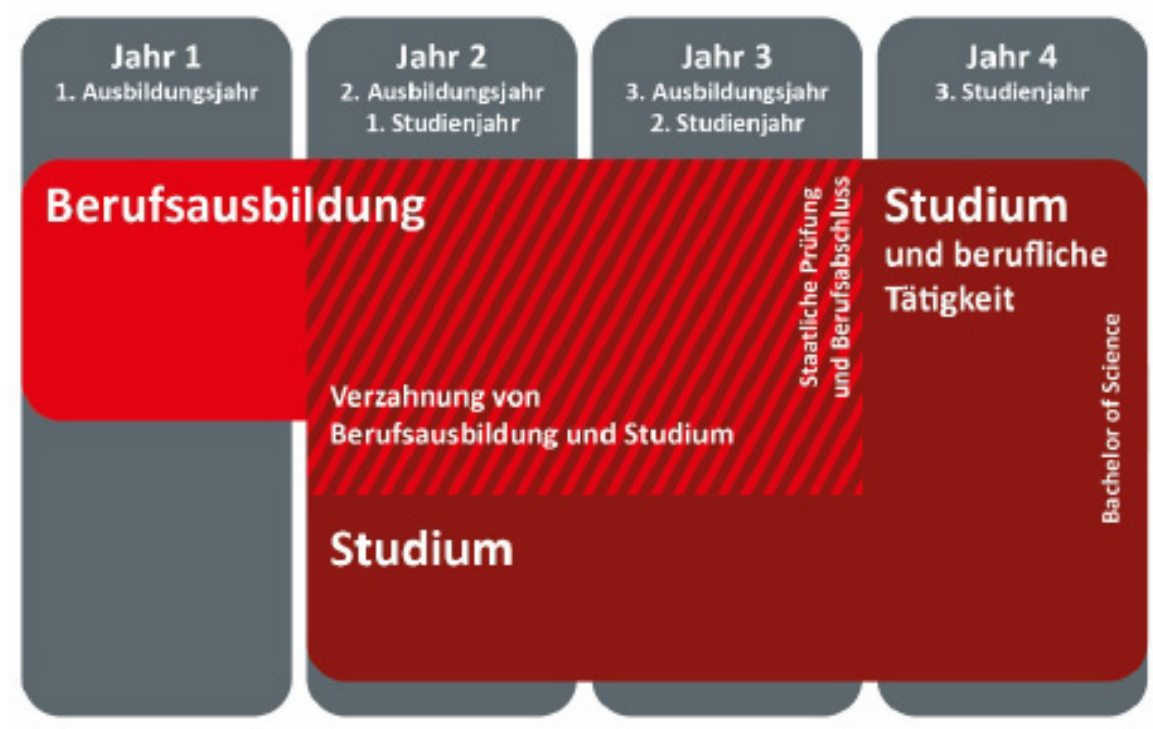

Abbildung 3: Das ausbildungsintegrierende Modell im Studienbereich Gesundheit der DHBW.

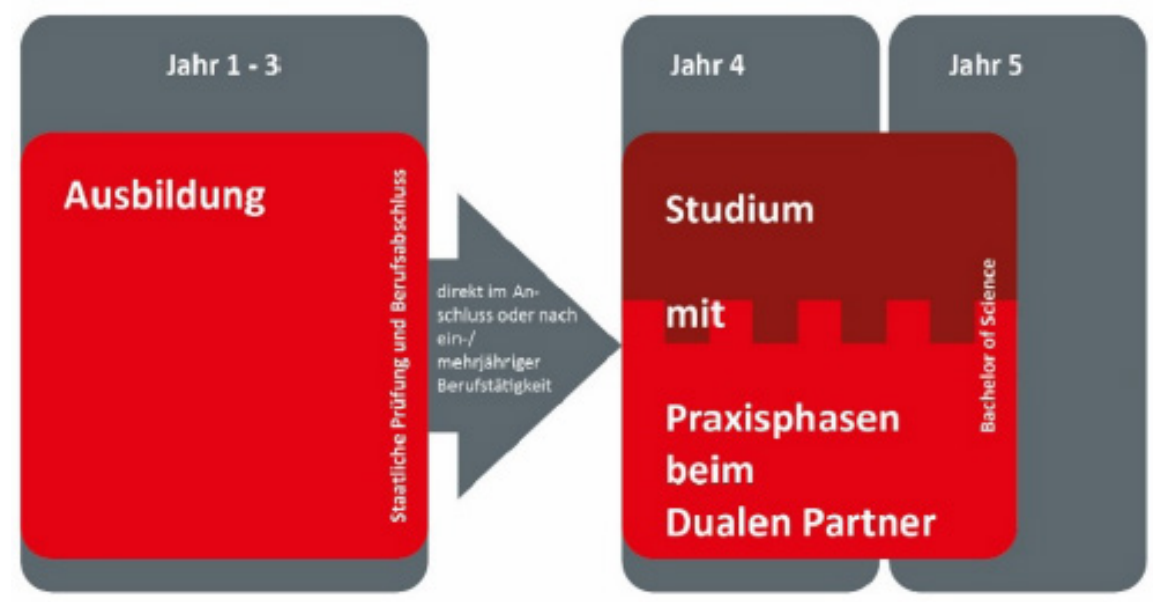

Abbildung 4: Das Anrechnungsmodell oder ausbildungsintegrierendes Modell I im Studienbereich Gesundheit der DHBW.

Geburtshilfe und Prävention. „Hebammen leisten einen unverzichtbaren Beitrag zur gesundheitlichen Versorgung von Frauen während der Schwangerschaft, bei der Geburt, während des Wochenbetts, in der Stillzeit und darüber hinaus sowie von Neugeborenen und Säuglingen.“ (BMG, 2019)

Somit soll das Hebammenstudium den Bedarf der flächendeckenden Versorgung von Frauen und Familien decken. Dieser besteht sowohl bei den Dualen Partnern als auch in allen Settings, die klinisch und außerklinisch Hebammenleistungen erfordern.

Den verantwortlichen Praxiseinrichtungen (gem. Hebammengesetz ausschließlich Krankenhäuser) kommen neben der vergüteten Durchführung des berufspraktischen Teils des Studiums besondere Aufgaben zu. Sie stellen die Praxiseinsätze mit einem hohen Anteil in der außerklinischen Versorgung bei freiberuflichen Hebammen oder in hebammengeleiteten Einrichtungen durch Kooperationsverträge sicher. Die Finanzierung der Kosten des praktischen Teils des Studiums erfolgt über das Krankenhausfinanzierungsreformgesetz (KHRG) durch die gesetzliche Krankenversicherung (GKV) und ist von der verantwortlichen Praxiseinrichtung zu verhandeln. Erste Erfahrungen zeigen einen hohen Bedarf der verantwortlichen Praxiseinrichtungen an Abstimmung, aber auch an Unterstützung durch die Hochschule bei der Entwicklung des Praxisstudiums. Die bestehende intensive Kooperation aus den langjährig durchgeführten ausbildungsintegrierenden Studienmodellen der DHBW und dementsprechend 


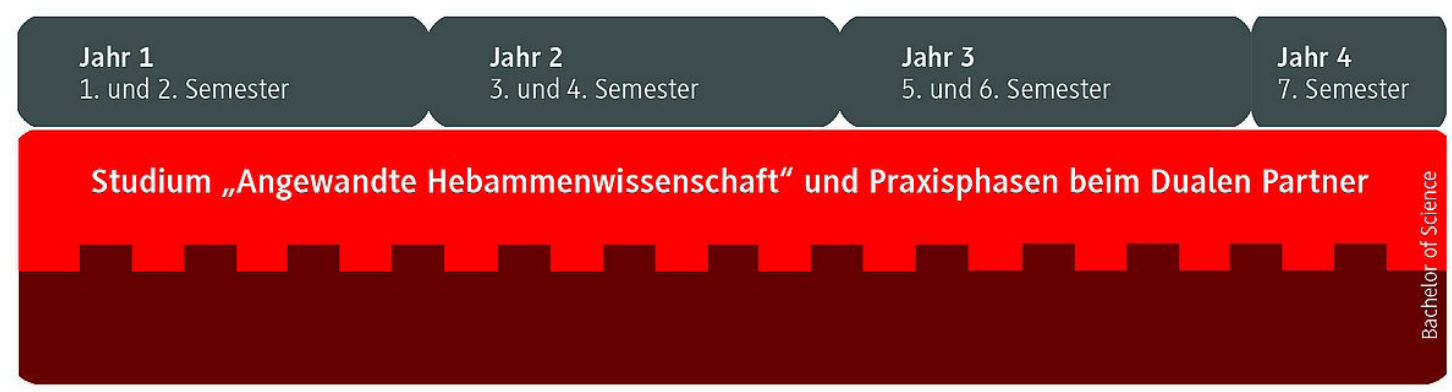

Abbildung 5: Das praxisintegrierende duale Kernmodell im primärqualifizierenden Hebammenstudium.

etablierte Kommunikationswege erleichtern den gemeinsamen Start in die Primärqualifizierung erheblich. Im Zuge der Reform des Hebammengesetzes wird derzeit der primärqualifizierende Studiengang an der DHBW entwickelt und nimmt im Oktober 2021 seinen Betrieb auf. Ab dem Jahr 2023 wird es die berufsfachschulische Ausbildung vollständig abgelöst haben. Das duale Studienmodell verbindet über sieben Semester in bewährter Weise die Theorie und Praxis im Hebammenstudium (s. Abb. 5).

\section{BESONDERE ROLLE DER DUALEN PARTNER}

DualePartnerimSinnederDHBW sindPraxiseinrichtungen, deren Eignung gemäß der Zulassungssatzung von den Studiengangsleitungen überprüft und vom örtlichen Hochschulrat festgestellt wird. Duale Partner können beispielsweise Pflegeeinrichtungen, Kliniken und Praxen sein. Sie müssen über mindestens 10 Mitarbeitende mit entsprechender Qualifikation verfügen, um die Qualität während der Praxisphasen kontinuierlich sicherstellen zu können. Duale Partner sind per Grundordnung Mitglied der Hochschule und in allen Gremien vertreten. Häufig sind nebenberuflich Lehrende Beschäftigte der Dualen Partner. Auch können bestimmte Lehrveranstaltungen, z. B. Praxisdemonstration oder Exkursionen, bei Dualen Partnern stattfinden.

Ohne Duale Partner ist das Modell der DHBW nicht umsetzbar. Am Anfang steht in diesem Modell der Bedarf der Dualen Partner für die Gesundheitsversorgung. Dementsprechend wählt der Duale Partner geeignete Mitarbeitende aus und delegiert sie zum Studium. Hierfür wird ein entsprechender Vertrag, der auch eine vom Ministerium vorgegebene, nicht rückzahlbare Mindestvergütung sowie Urlaub regelt, zwischen Dualem Partner und Studierenden geschlossen. Dieser Vertrag ist neben den üblichen Hochschulzulassungskriterien die entscheidende Grundvoraussetzung zum Studium(DHBW 2018). Die DHBW nimmt keine Bewerbungen auf einen Studienplatz an. Die Auswahl geeigneter Bewerber/innen obliegt dem Dualen Partner, der ausgewählten
Mitarbeitenden zum Studium an der jeweiligen Studienakademie anmeldet. Damit hat der Duale Partner die Auswahlfreiheit und gleichzeitig die Möglichkeit, die lebenszyklusorientierte Personalentwicklung der Mitarbeitenden entsprechend vorzunehmen. Auch junge Einsteigerinnen und Einsteiger haben in den großen Praxisphasen über die Hälfte des Studiums hinweg die Möglichkeit, die Einrichtung kennenzulernen und durch die wissenschaftlichen Arbeiten im Auftrag der Einrichtung mitzugestalten.

Duale Partner sind verpflichtet, ihre Eignung nachzuweisen, hierzu erforderliche Unterlagen zur Verfügung zu stellen und Besichtigungen vor Ort zu ermöglichen. Zum Erreichen des Ausbildungsziels nach dem Rahmenstudienplan des Studiengangs vermittelt der Duale Partner die erforderlichen Kenntnisse, Fertigkeiten und beruflichen Erfahrungen. Grundlage bildet hierfür der personalisierte Ausbildungsplan, der in Verantwortung einer autorisierten Person (Ausbildungsleiterin, Ausbildungsleiter) erstellt und ggf. in Zusammenarbeit mit Ausbilderinnen, Ausbildern, Anleiterinnen, Anleitern (z. B. in einer anderen Abteilung oder Filiale) umgesetzt wird. Mittel zur praktischen Ausbildung (z. B. Fachliteratur) sowie Arbeitskleidung muss den Studierenden vom Dualen Partner zur Verfügung gestellt werden. Organisatorisch ist der Duale Partner an den Rahmenstudienplan gebunden, d. h., er muss den Studierenden für die dreimonatigen Theoriephasen freistellen und hat den Urlaub so zu gewähren, dass der Studierende diesen innerhalb der Praxisphasen vollständig antreten kann. Außerdem muss der Duale Partner den Studierenden ausreichend Gelegenheit zur Anfertigung von Prüfungsleistungen einräumen. In der Regel wird die Themenvergabe wissenschaftlicher Arbeiten, insbesondere der Bachelor-Arbeit, gemeinsam zwischen Dualem Partner und der Studiengangsleitung, die das Thema genehmigt, abgestimmt.

In Sektoren, wie der Physio- und Ergotherapie in der direkten Patientenversorgung, ist der Bedarf akademisch qualifizierter Mitarbeitender in Praxen, Kliniken und Krankenhäuser auch durch die gesetzgeberische 
Steuerung mittels Abrechnungsziffern vergleichsweise geringer als im Bereich der Pflege, der Geburtshilfe/ Hebammenkunde oder im Management. Die Ausbildung im Therapiebereich war traditionell an Berufsfachschulen angesiedelt, wobei die Praxiseinrichtungen die Ausbildung nicht vergüten. Der mit der Akademisierung verbundene Nutzen für die Praxiseinrichtung und Gesellschaft konnte noch zu wenig gezeigt werden. Daher ist es in diesen Studiengängen der DHBW eine besondere Herausforderung, Duale Partner mit entsprechender Personalstrategie und Risikobereitschaft zu rekrutieren.

\section{BESONDERE ROLLE DER STUDIERENDEN}

Die Studierenden sind Mitarbeitende der Dualen Partner und nehmen aus dieser Stellung heraus eine besondere Rolle ein. Sie sind insofern Botschafterinnen und Botschafter sowie Weiterentwicklerinnen und Weiterentwickler sowohl für den Dualen Partner als auch die DHBW. Darüber hinaus wird die allgemein als sehr frei eingeschätzte „Studentenzeit" so nicht erlebt. Die Studierenden haben durch die Delegation und aus dem Studienvertrag heraus eine Anwesenheitspflicht im Studium. Das Studium ist stark getaktet und spiegelt einen hohen Anspruch aus dem Qualitätsniveau der Einrichtung und aus dem Qualifikationsrahmen (DQR6-Niveau) wider. Das Intensivstudium bedeutet eine Nettojahresarbeitszeit von 2100 Stunden. Nebenjobs sind in aller Regel aus Zeitgründen nicht möglich. Das gilt auch für die Tätigkeit als Studentische Hilfskraft und die damit möglicherweise frühzeitige Anbahnung von wissenschaftlichen Karrieren.

Dieser spezifische Nachteil wird insofern kompensiert, als dass die Studierenden mit Beginn ihres Studiums bereits beim Dualen Partner wissenschaftliche Arbeiten realisieren, die dann in der Bachelor-Arbeit gipfeln. Zudem können sie in kooperativen Forschungsprojekten zwischen Dualem Partner und der DHBW mitwirken. Die Bachelor-Abschlüsse sind anschlussfähig. Über das Center of Advanced Studies (CAS) der DHBW werden kooperative Master-Programme angeboten, die speziell im Gesundheitsbereich und interprofessionell entwickelt wurden (Flaiz et al., 2019). Darüber hinaus können Studierende im Rahmen von kooperativen Promotionen ihre wissenschaftliche Karriere fortsetzen. Gleichermaßen ist die Anschlussfähigkeit gegeben und es stehen den DHBW-Absolventinnen und Absolventen grundsätzlich alle Master- und Promotionsprogramme anderer Hochschulen und Universitäten, auch international, offen.

Für Studierende im DHBW-Modell, insbesondere in den therapiebezogenen Gesundheitsstudiengängen, ist die Pilotrolle derzeit noch stark herausfordernd. In der therapeutischen Ausbildung ist berufliches
Bildungspersonal analog der Praxisanleitung in der Pflege vom Gesetzgeber nicht verlangt und daher in den Einrichtungen auch nicht explizit verfügbar. Als Brücke werden in den Einrichtungen Projekte mit dem Ziel initiiert, konkrete Aufgaben mit akademischem Anspruch und entsprechende Anleitungskonzepte $\mathrm{zu}$ definieren (Winkelmann et al., 2019).

\section{BESONDERE ROLLE DER STUDIENGANGSLEITUNG}

Neben den Dualen Partnern ist die Studiengangsleitung im Modell der DHBW und zur Realisierung des Intensivstudiums unverzichtbar (vgl. Abschnitt „Das Original“). Sie hat die Rolle einer Managerin oder eines Managers inne und stellt als Instanz eine Art Netz-KnotenPunkt dar (s. Abb. 6). Der Studiengang gilt als kleinste geschlossene Organisationseinheit der DHBW. Im Vergleich zu anderen hochschulischen und universitären Strukturen und Prozessen sind neben organisatorischen Aufgaben der Studiengangsleitung die Gewinnung von und die nachhaltige Kontaktpflege zu Dualen Partnern als Unterscheidungsmerkmal zu nennen.

\section{FORSCHUNG UND WISSENSCHAFTLICHE WEITERENTWICKLUNG AN DER DHBW}

Dem Anspruch des Konzeptes der DHBW nach Verzahnung von Theorie und Praxis folgend, realisiert die DHBW auch in Bezug auf die Forschung und Entwicklung einen spezifischen Forschungsauftrag als anwendungs- und praxisorientierte sowie kooperative Forschung mit den Dualen Partnern. Ziel der Forschungsförderlinie des Wissenschaftsministeriums in Baden-Württemberg ist es dabei, in enger Kooperation mit den Dualen Partnern den Bereich Forschung anwendungs- und transferorientiert neben der Lehre zu etablieren (Ministerium für Wissenschaft, Forschung und Kunst Baden-Württemberg (MWK, o. J.). Im Zentrum stehen Forschungsfragen und Untersuchungsgegenstände zur Entwicklung innovativer Konzepte, Strategien und Technologien für die konkreten Bedingungen in Wirtschaft, Technik, Sozialwesen und Gesundheit. Die zu schaffende Forschungsinfrastruktur soll kooperative Forschungsansätze mit den Dualen Partnern, mit weiteren Studienakademien der DHBW (Verbundforschung) sowie mit Partnern auf nationaler und internationaler Ebene (z. B. als virtueller Campus Gesundheit) ermöglichen. Mit der sich so entwickelnden Forschungskultur an der DHBW werden die Studienkonzepte, der Transfer und der internationale Austausch gestärkt. Die DHBW nimmt erfolgreich am Programm des Bundes zur Ermöglichung von Kooperativen Promotionen an außeruniversitären Hochschuleinrichtungen teil. Sie fördert damit den 


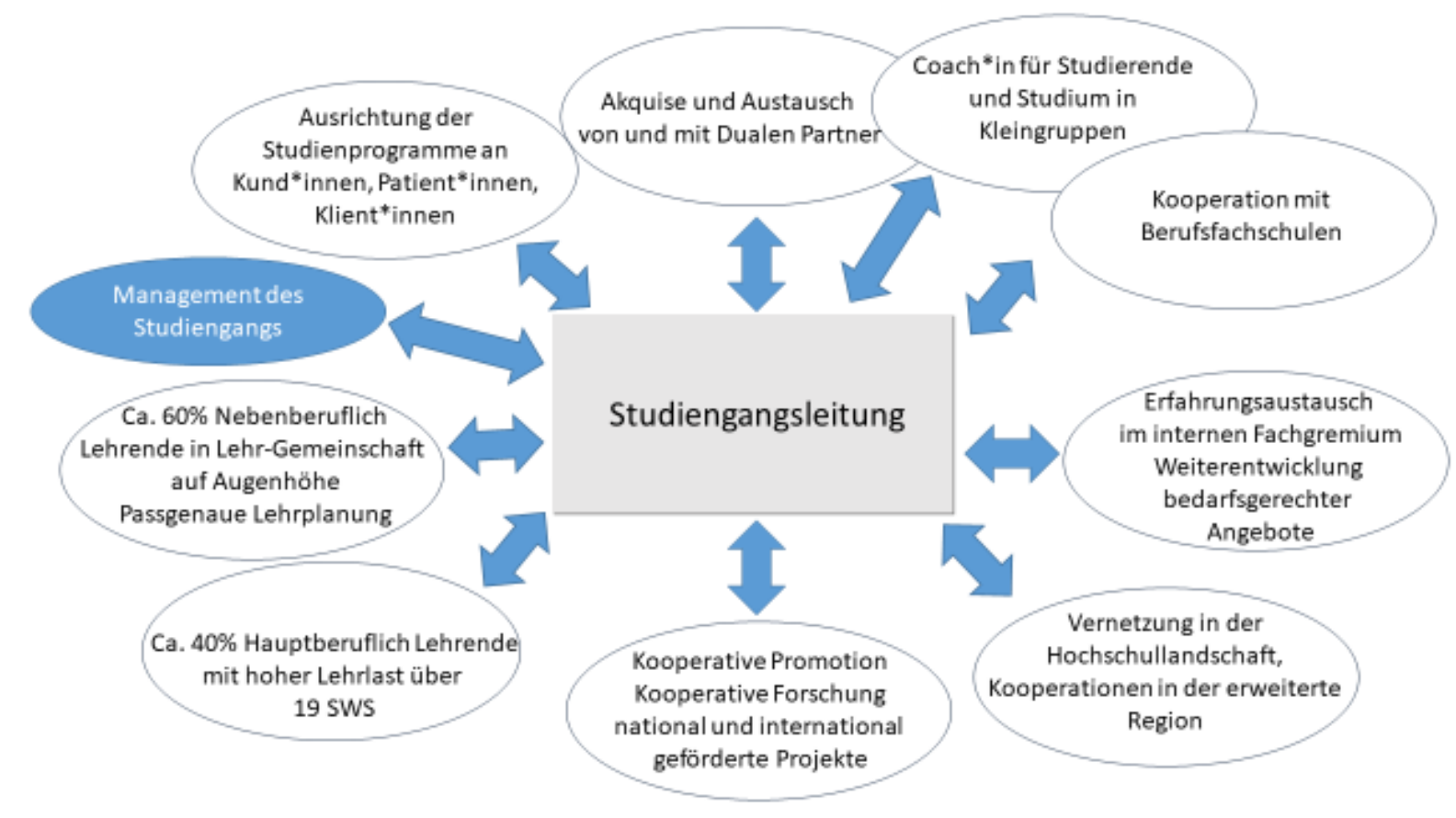

Abbildung 6: Komplexes Aufgabenfeld der Studiengangsleitung an der DHBW.

wissenschaftlichen Nachwuchs und etablierte ein eigenes Kooperatives Promotionsprogramm mit ca. 30 Promotionsplätzen alle drei Jahre.

\section{TAKE HOME}

Die DHBW bietet aus einer seit mehr als 50 Jahren gelebten Tradition von Verschränkung der Theorie mit der Praxis erfolgreich und nachhaltig Studienprogramme auf Bachelor- und Master-Niveau in den Bereichen „Wirtschaft“, „Technik“, „Sozialwesen“ und "Gesundheit“ an. Ebenso kooperativ erfolgen die Forschung und Entwicklung. Das BachelorStudium der DHBW ist als Intensivstudium mit einem Dualen Kernmodell akkreditiert. Für den Bereich Gesundheit existiert ein spezifisches Rahmenmodell mit diversen Studienformaten, um den Besonderheiten und Vorgaben in diesem Bereich entsprechen zu können. Das Duale Kernmodell dient mittlerweile national als auch international anderen Hochschulen als Referenz. Der nachhaltige Erfolg setzt ein Denken und Handeln, ausgehend vom Bedarf der Gesellschaft, voraus und eine dementsprechende interne und externe Infrastrukturlandschaft der Hochschule.

Literatur

BMG (2019). Gesundheitsberufe. Hebammen. https://www. bundesgesundheitsministerium.de/themen/gesundheitswesen/ gesundheitsberufe/hebammen.html, abgerufen am 16.03.2021.

DHBW (2018). Studienvertrag. https://www.dhbw.de/fileadmin/user_ upload/Dokumente/Dokumente_fuer_Duale_Partner/DHBW_ Studienvertrag.pdf, abgerufen am 11.03.2021.

DHBW (2021). Duale Hochschule Baden-Württemberg. DHBW. https://www.dhbw.de, abgerufen am 11.03.2021.

Flaiz, B., Winkelmann, C., \& Simon, A. (2019). On the Development of the Competence Profile for the Master Program "Advanced Practice in Health Care". Application-Oriented Higher Education Research (AOHER) Vol 4 No 4 77-84, CN34-1326/G4.
HebG (2021). Gesetz über das Studium und den Beruf von Hebammen (Hebammengesetz - HebG) vom 22. November 2019 (BGB1. I S. 1759), das durch Artikel 10 des Gesetzes vom 24. Februar 2021 (BGBl. I S. 274) geändert worden ist.

LHG (2020). Gesetz über die Hochschulen in Baden-Württemberg (Landeshochschulgesetz - LHG), §2, Absatz 1, Satz 5. http://www. landesrecht-bw.de/jportal/portal/t/did/page/bsbawueprod.psml?doc. hl=1\&doc.id=jlr-HSchulGBWV28IVZ\&documentnumber=2\&num berofresults $=121 \&$ doctyp $=$ Norm \&showdoccase $=1 \&$ doc.part $=$ S \&pa ramfromHL=true\#focuspoint, abgerufen am 11.03.2021.

MWK (o. J.). Innovative Konzepte. Kooperative Forschung an der Dualen Hochschule Baden-Württemberg. https://mwk. 
baden-Swuerttemberg.de/de/forschung/forschungsfoerderung/ technologietransfer/kooperative-forschung-an-der-dualenhochschule-baden-wuerttemberg/, abgerufen am 11.03.2021.

Winkelmann, C., \& Lüderitz, C. (2019). Lernen im hochschulischen Praktikum - Qualifizierung von Mentorinnen und Mentoren in Gesundheitseinrichtungen. In: Siegmüller, J., \& Winny, S. (Hrsg.) Professionalisierung durch Akademisierung. Hochschulbildung in den therapeutischen Berufen. Dr. Kovac. 215-232. ISSN 21943753.

WR (2013): Empfehlungen zur Entwicklung des dualen Studiums - Positionspapier. https://www.wissenschaftsrat.de/download/ archiv/3479-13.pdf, abgerufen am 20.02.2021. 